\title{
Phagocytosis of particulate antigens - all roads lead to calcineurin/NFAT signaling pathway
}

\author{
Jan Fric, Teresa Zelante and Paola Ricciardi-Castagnoli * \\ Singapore Immunology Network (SIgN), Agency for Science, Technology and Research (A*STAR), Biopolis, Singapore \\ ${ }^{*}$ Correspondence: paola_castagnoli@immunol.a-star.edu.sg \\ Edited by: \\ Fabrizio Mattei, Istituto Superiore di Sanità, Italy
}

Keywords: NFAT, dendritic cells, innate immunity, calcineurin, TLRs, phagocytosis, dectin-1 receptors, marco

Antigen-presenting cells (APC) possess multiple cell surface receptors that recognize common microbe-associated antigens as well as immune complexes and inert particles. Upon encountering such antigens these receptors must cooperate to achieve phagocytosis and trigger signaling cascades that initiate the innate immune response. While the stimuli initiating these signaling cascades are diverse, recent data have revealed that their effects in APC and particularly in dendritic cells (DC), all have something in common: downstream activation of nuclear factor of activated T cells (NFAT). NFAT is a family of transcription factors that has emerged as a key mediator of the initiation of immune responses by APCs, and specifically of IL-2 production by DC as reviewed (1). Intriguingly NFAT activation now seems to be the shared endpoint of several signaling pathways that all begin with uptake of particulate antigens.

Notably, the NFAT family members appeared at the origin of vertebrates whereas nearly all the other signaling pathways, including NFKB pathway, are very ancient and present in all invertebrate's species. NFAT signaling plays essential roles in vertebrate organogenesis and development but also in the formation of adaptive immunity. In addition, G. R. Crabtree has suggested that "NFAT may have contributed to the evolutionary adaptation of innate immunity: e.g., minimize the costs of inflammation by collaboration with adaptive immunity" (2). It is likely that the ability of DC to link innate with adaptive immunity might be the result of DC's ability to couple phagocytic functions to NFAT activation, leading to extensive gene reprograming.

This is the latest in a series of new hypothesis to better understand of the true complexity of the process of pathogen sensing, uptake, and response in APC and in particular in DC. But as is so often the case, with new hypotheses and knowledge has come new questions: how can such diverse stimuli all converge on similar pathways of immune activation? How do APCs integrate signaling from multiple immune uptake receptors? And how can we explain the difference in APC responses to soluble and particulate antigens?

In this article we will review the recent steps forward in our understanding of the intricate cross-talk between pathways of phagocytosis and immune signaling in APC, and the evidence that NFAT activation is a unique hallmark of this process.

\section{PHAGOCYTIC RECEPTORS AS MASTER REGULATORS OF UPTAKE}

Pattern recognition receptors (PRRs) are expressed abundantly by APCs, both on the cell surface and in intracellular compartments, and are ligated by conserved microbe-associated molecular patterns (MAMPs). Signaling by PRRs is important for innate immune cell activation, maturation, antigen processing, and presentation, and it now seems to be influenced by the process of phagocytosis itself. For example, it has long been known that responses to soluble and particulate forms of the same MAMP can vary enormously, but it is only recently that the interaction of phagocytosis and PRRs has been implicated in the mechanism of this distinction.

Phagocytosis and MAMP detection by APC have often been considered as complimentary but separate processes; however, it was noted that during microbe uptake, certain cell surface PRRs were actively recruited to the forming endosomes or phagosomes together with the microbial load. This subset of PRRs, including C-type lectin receptors and CD14, was termed the
"Phagocytic Receptors"(3), and has since been extended to include opsonic receptors such as Fc receptors, which mediate uptake of immune complexes. However, it now seems that Phagocytic Receptors do not only mediate uptake of particulate antigens, but can also determine the recruitment, activation, and intracellular signaling of other PRRs during the process of phagocytosis and antigen/pathogen degradation (4).

\section{PHAGOCYTIC RECEPTORS CO-ORDINATE COMPLEX IMMUNE RESPONSES TO SIMPLE MAMPS}

Lipopolysaccharides (LPS) are highly immunogenic MAMPs on Gram-negative bacteria, and are recognized by several receptors on the APC surface; CD14, for example, is well-established as a nonopsonic Phagocytic Receptor for bacteria (5), but has recently been found to mediate the internalization of another LPS receptor, TLR4, via signaling through the tyrosine kinase Syk and PLC $\gamma_{2}$ (6). What is particularly interesting about TLR4 is the effect that this process of internalization has upon the resulting signaling pathways that are triggered. Ligation of TLR4 by LPS at the plasma membrane activates the adaptor proteins TIRAP and MYD88, which enable pro-inflammatory gene transcription. However, once internalized with its ligand, TLR4 instead promotes TRAM-TRIF signaling, culminating in the production of type I IFNs $(7,8)$. Thus CD14 controls TLR4 internalization, and specific adaptor localization controls the downstream signaling pathways that result.

Alongside its interaction with TLR4, CD14 is also required for LPS signaling through Src-family kinases and PLC $_{\gamma 2}$ activation. As shown in murine DC this leads to $\mathrm{Ca}^{2+}$ flux and the activation of 
the phosphatase calcineurin, which causes NFAT nuclear translocation and so the release of IL-2 (9-11).

\section{PHAGOCYTIC RECEPTORS FACILITATE DISTINCTION OF SOLUBLE AND PARTICULATE MAMPS}

Whole microbes are significantly more potent immune stimulators than the soluble versions of their MAMPs, but understanding how APCs sense this difference has been challenging. Substantial progress was made recently when a study exposing murine DC to either soluble or beadbound LPS revealed stronger triggering of Syk/PLC $\gamma_{2}$ in response to "particulate" LPS (6), possibly resulting in increased $\mathrm{Ca}^{2+}$ influx-dependent NFAT activation and transcription of downstream targets. This hinted at the importance of the NFAT pathway in determining immune outcomes to particulate antigens, however it was an elegant set of studies on the C-type lectin receptor, Dectin-1, that illustrated a molecular mechanism underlying APC detection of MAMP forms (12).

Dectin-1 is a non-opsonic Phagocytic Receptor that recognizes the major component of the outer fungal cell wall, 1,3$\beta$ glucan (13-15). Upon ligand binding and receptor dimerization, the hemiITAM motif in the cytoplasmic tail of Dectin-1 is phosphorylated by Src-family kinases, which enables recruitment and activation of Syk. In turn, Syk mediates the MAPK response, canonical and noncanonical NFKB activation and also, importantly, stimulates the $\mathrm{Ca}^{2+}$ flux that drives calcineurin dephosphorylation of NFAT, leading to its nuclear translocation (15). This NFAT signaling is clearly linked with the particulate form of $1,3-\beta$ glucan and phagocytosis (Figure 1) and inducing different cytokine pattern than soluble form (Figure 1).

What has now emerged is that the differences in this process following ligation by soluble versus particulate ligands enable receptor-driven distinction between the two forms of the same antigen. The soluble $\beta-1,3 / \beta-1,6$-linked glucans isolated from the cell wall of Saccharomyces bind single molecules of Dectin-1 and induce hemi-ITAM phosphorylation by Src-family kinases, but downstream signaling events, including calcium flux, are limited and transient. This results from

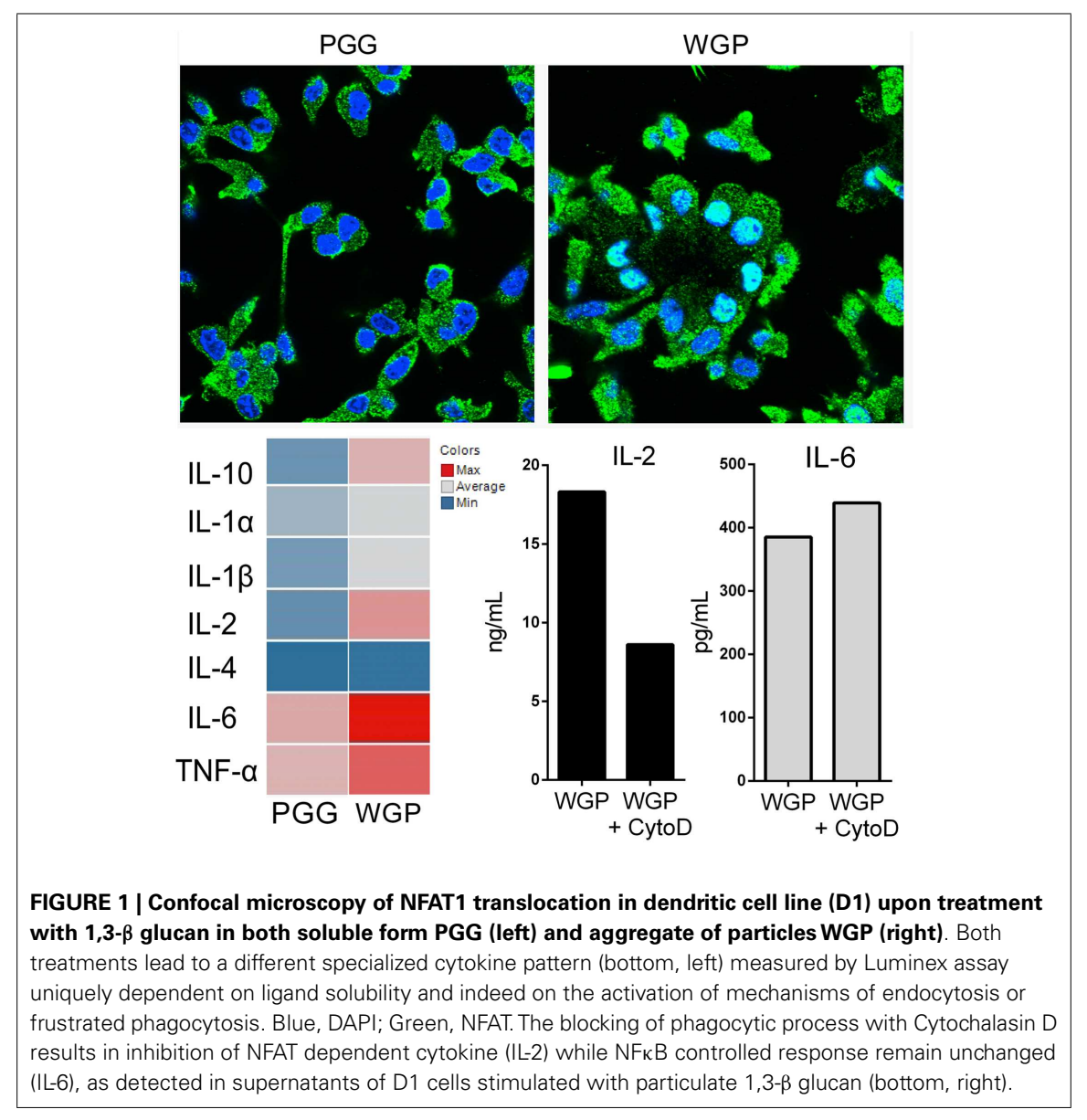

expression of the membrane phosphatases CD45 and CD148 alongside Dectin-1, which serve to de-phosphorylate the Src kinases thereby impeding effective Dectin1 signaling (12). In contrast, the aggregation of the $\beta-1,3$ glucan ligand on the surface of a yeast enables a parallel aggregation of Dectin-1 on the APC surface which increases receptor avidity and results in the formation of a "phagocytic synapse." The close physical arrangement of ligands and receptors in the synapse excludes CD45 and CD148, releasing the brakes on sustained Src activation, and enabling induction of the cytoskeletal rearrangements required for phagocytosis, as well as the sustained calcium flux that is linked to NFAT activation and initiation of transcription of NFAT dependent cytokines (Figure 1).

\section{PHAGOCYTIC RECEPTORS DELIVER EXTRACELLULAR MAMPS TO INTRACELLULAR PRRS}

The macrophage receptor with collagenous structure (MARCO) (16) is a non-opsonic multi-ligand Phagocytic Receptor whose function remained an enigma for many years; while MARCO is implicated in the pathogenesis of many inflammatory diseases, it is completely unable to initiate proinflammatory signaling itself (17). Several studies now indicate that it is MARCO's interactions with other PRRs that define its role in shaping responses to particulate antigens. For example, strong and sustained signaling through the intracellular PRR TLR3 in response to the ligand PolyIC, required MARCO for rapid delivery and concentration of the ligand in the phagosome (18). In the case of the mycobacterial cell wall glycolipid trehalose 6,69-dimycolate, MARCO serves to enhance signaling trough the TLR2/CD14 complex (Bowdish PlosOne, 2009), where both molecules are known to be able to induce NFAT activation (10, 15). Indeed, MARCO-mediated phagocytosis of sterile particulates such as silica is the first step in a signaling cascade ending in NFAT activation and TNF transcription 
$(19,20)$. Cytoskeleton rearrangement has been observed as a result of MARCO clustering in activated DC (21).

\section{PHAGOCYTIC RECEPTORS LINK ANTIGEN UPTAKE TO PRR ACTIVATION}

Fc $\gamma$ Rs are members of the immunoglobulin superfamily and are opsonic Phagocytic Receptors that recognize the Fc portion of antibodies bound to their cognate antigen. This process is central to the clearance of pathogens during infection, but has also been well studied for its role in autoimmune disease: in systemic lupus erythematosus (SLE) large self-DNAcontaining immune complexes (DNA-ICs) are internalized by Fc $\gamma$ Rs on the surface of DC that then produce pro-inflammatory cytokines which contribute to disease pathology $(22,23)$. Signaling via the $F c \gamma R$ is integral to both these processes; DNA-IC binding to the $\mathrm{Fc} \gamma \mathrm{R}$ induces its phagocytosis and also triggers activation of Src-family kinases, which first drive the cytoskeletal rearrangements needed to recruit the DNA-sensing TLR9 to the phagosome, and subsequently mediate phosphorylation of TLR9 (23). TLR9 activation in turn results in the recruitment and activation of the kinase Syk, and also activates the adaptor MYD88 which induces NFkBmediated cytokine gene transcription and the secretion of type I IFNs. Whether Fc $\gamma$ Rmediated uptake and immune activation specifically link with NFAT nuclear translocation has not yet been assessed, though the central role of activated Syk is suggestive (24). However, the related Phagocytic Receptor FceRI (25), does trigger substantial calcium flux and NFAT translocation following ligand binding (26-28).

\section{CONCLUDING REMARIS}

It is increasingly evident that the processes of immune uptake and immune signaling can no longer be considered as discrete, but rather are highly integrated by APC in order to induce multiple and inter-linked signaling pathways. One aspect of this is the roles of Phagocytic Receptors, some of which have been highlighted here. Despite diverse molecular structures and varied ligands, members of the Phagocytic Receptor family share the ability to direct downstream signaling toward triggering calcium influx, followed by calcineurin activation and NFAT translocation. NFAT signaling has been studied within myeloid cells (11, 29). Here we propose that the activation of calcineurin-NFAT signaling might be considered a hallmark of successful initiation of early innate responses toward phagocytosed particulate antigens. Interestingly, there is now evidence that NFAT activation may be part of a multi-component signature of the APC response to particulates; a recent study has shown that a common characteristic of the phagosomes formed during uptake by Fc $\gamma$ Rs, MARCO, and Dectin- 1 is the accumulation of the autophagy marker LC3 (30). While the full implication of this observation remains to be investigated, the presence of LC3 implies a degree of cross-talk between pathways of particulate uptake and the non-canonical autophagic response of cells to ligands likely to include DNA-IC, Mycobacteria, and fungi.

These advances in understanding of the Phagocytic Receptors are already beginning to pay dividends; MARCO-mediated uptake of antigen-coated microparticles has successfully been exploited to ameliorate disease in a murine model of autoimmune encephalomyelitis (EAE) (31), while particles coated in the Dectin-1 ligands $\beta$ 1,3-linked and $\beta$-1,6-linked glucans have been used as a potent vaccine delivery system in mice (32). Further understanding of the fine-tuned mechanisms underlying particulate uptake and immune signaling in APC, and the ensuing innate outcomes, e.g., the production of regulatory cytokines by DC such as IL-2 and IL-23 (1) has significant potential to improve our ability to design effective vaccines against infectious diseases and for the treatment of autoimmune conditions.

\section{ACKNOWLEDGMENTS}

This research was funded by SIgN, A*STAR, Singapore. The authors wish to thank Lucy Robinson of Insight Editing London for assistance in manuscript preparation.

\section{REFERENCES}

1. Zelante T, Fric J, Wong AY, Ricciardi-Castagnoli P. Interleukin-2 production by dendritic cells and its immuno-regulatory functions. Front Immunol (2012) 3:161. doi:10.3389/fimmu.2012.00161

2. Wu H, Peisley A, Graef IA, Crabtree GR. NFAT signaling and the invention of vertebrates. Trends Cell Biol (2007) 17:251-60. doi:10.1016/j.tcb.2007.04. 006
3. Jaumouille V, Grinstein S. Receptor mobility, the cytoskeleton, and particle binding during phagocytosis. Curr Opin Cell Biol (2011) 23:22-9. doi:10. 1016/j.ceb.2010.10.006

4. Barton G, Kagan J. A cell biological view of Toll-like receptor function: regulation through compartmentalization. Nat Rev Immunol (2009) 9:535-42. doi:10.1038/nri2587

5. Schiff DE, Kline L, Soldau K, Lee JD, Pugin J, Tobias PS, et al. Phagocytosis of gram-negative bacteria by a unique CD14-dependent mechanism. J Leukoc Biol (1997) 62:786-94.

6. Zanoni I, Ostuni R, Marek L, Barresi S, Barbalat $\mathrm{R}$, Barton G, et al. CD14 controls the LPS-induced endocytosis of Toll-like receptor 4. Cell (2011) 147:868-80. doi:10.1016/j.cell.2011.09.051

7. Hagar J, Powell D, Aachoui Y, Ernst R, Miao E. Cytoplasmic LPS activates caspase-11: implications in TLR4-independent endotoxic shock. Science (2013) 341:1250-3. doi:10.1126/science. 1240988

8. Kayagaki N, Wong M, Stowe I, Ramani S, Gonzalez L, Akashi-Takamura S, et al. Noncanonical inflammasome activation by intracellular LPS independent of TLR4. Science (2013) 341:1246-9. doi:10.1126/science. 1240248

9. Granucci F, Vizzardelli C, Pavelka N, Feau S, Persico M, Virzi E, et al. Inducible IL-2 production by dendritic cells revealed by global gene expression analysis. Nat Immunol (2001) 2:882-8. doi:10. 1038/ni0901-882

10. Zanoni I, Ostuni R, Capuano G, Collini M, Caccia $\mathrm{M}$, Ronchi AE, et al. CD14 regulates the dendritic cell life cycle after LPS exposure through NFAT activation. Nature (2009) 460:264-8. doi:10.1038/ nature 08118

11. Fric J, Zelante T, Wong AY, Mertes A, Yu HB, Ricciardi-Castagnoli P. NFAT control of innate immunity. Blood (2012) 120:1380-9. doi:10.1182/ blood-2012-02-404475

12. Goodridge HS, Underhill DM, Touret N. Mechanisms of Fc receptor and dectin-1 activation for phagocytosis. Traffic (2012) 13:1062-71. doi:10. 1111/j.1600-0854.2012.01382.x

13. Brown GD, Gordon S. Immune recognition. A new receptor for beta-glucans. Nature (2001) 413:36-7. doi:10.1038/35092620

14. Brown GD. Dectin-1: a signalling non-TLR pattern-recognition receptor. Nat Rev Immunol (2006) 6:33-43. doi:10.1038/nri1745

15. Goodridge HS, Simmons RM, Underhill DM. Dectin-1 stimulation by Candida albicans yeast or zymosan triggers NFAT activation in macrophages and dendritic cells. J Immunol (2007) 178: 3107-15.

16. van der Laan LJ, Dopp EA, Haworth R, Pikkarainen $\mathrm{T}$, Kangas M, Elomaa O, et al. Regulation and functional involvement of macrophage scavenger receptor MARCO in clearance of bacteria in vivo. J Immunol (1999) 162:939-47.

17. Peiser L, De Winther MP, Makepeace K, Hollinshead M, Coull P, Plested J, et al. The class A macrophage scavenger receptor is a major pattern recognition receptor for Neisseria meningitidis which is independent of lipopolysaccharide and not required for secretory responses. Infect Immun (2002) 70:5346-54. doi:10.1128/IAI.70.10. 5346-5354.2002 
18. Mukhopadhyay S, Varin A, Chen Y, Liu B, Tryggvason K, Gordon S. SR-A/MARCO-mediated ligand delivery enhances intracellular TLR and NLR function, but ligand scavenging from cell surface limits TLR4 response to pathogens. Blood (2011) 117:1319-28. doi:10.1182/blood-2010-03276733

19. Ke Q, Li J, Ding J, Ding M, Wang L, Liu B, et al. Essential role of ROS-mediated NFAT activation in TNF-alpha induction by crystalline silica exposure. Am J Physiol Lung Cell Mol Physiol (2006) 291:L257-64. doi:10.1152/ajplung.00007.2006

20. Thakur SA, Beamer CA, Migliaccio CT, Holian A. Critical role of MARCO in crystalline silicainduced pulmonary inflammation. Toxicol Sci (2009) 108:462-71. doi:10.1093/toxsci/kfp011

21. Granucci F, Petralia F, Urbano M, Citterio S, Di Tota F, Santambrogio L, et al. The scavenger receptor MARCO mediates cytoskeleton rearrangements in dendritic cells and microglia. Blood (2003) 102:2940-7. doi:10.1182/blood-2002-12-3651

22. Means TK, Latz E, Hayashi F, Murali MR, Golenbock DT, Luster AD. Human lupus autoantibodyDNA complexes activate DCs through cooperation of CD32 and TLR9. J Clin Invest (2005) 115:407-17. doi:10.1172/JCI200523025

23. Sanjuan MA, Rao N, Lai KT, Gu Y, Sun S, Fuchs A, et al. CpG-induced tyrosine phosphorylation occurs via a TLR9-independent mechanism and is required for cytokine secretion. J Cell Biol (2006) 172:1057-68. doi:10.1083/jcb.200508058

24. Sedlik C, Orbach D, Veron P, Schweighoffer E, Colucci F, Gamberale R, et al. A critical role for Syk protein tyrosine kinase in Fc receptormediated antigen presentation and induction of dendritic cell maturation. J Immunol (2003) 170: 846-52.

25. Maurer D, Fiebiger E, Reininger B, Wolff-Winiski B, Jouvin MH, Kilgus O, et al. Expression of functional high affinity immunoglobulin $\mathrm{E}$ receptors (Fc epsilon RI) on monocytes of atopic individuals. J Exp Med (1994) 179:745-50. doi:10.1084/ jem.179.2.745

26. Kitaura J, Xiao W, Maeda-Yamamoto M, Kawakami Y, Lowell CA, Kawakami T. Early divergence of Fc epsilon receptor I signals for receptor upregulation and internalization from degranulation, cytokine production, and survival. J Immunol (2004) 173:4317-23.

27. Klein M, Klein-Hessling S, Palmetshofer A, Serfling E, Tertilt C, Bopp T, et al. Specific and redundant roles for NFAT transcription factors in the expression of mast cell-derived cytokines. I Immunol (2006) 177:6667-74.

28. Walczak-Drzewiecka A, Ratajewski M, Wagner W, Dastych J. HIF-1alpha is up-regulated in activated mast cells by a process that involves calcineurin and NFAT. J Immunol (2008) 181: 1665-72.

29. Fric J, Lim CX, Koh EG, Hofmann B, Chen J, Tay HS, et al. Calcineurin/NFAT signalling inhibits myeloid haematopoiesis. EMBO Mol Med (2012) 4:269-82. doi:10.1002/emmm.201100207

30. Bonilla DL, Bhattacharya A, Sha Y, Xu Y, Xiang Q, Kan A, et al. Autophagy regulates phagocytosis by modulating the expression of scavenger receptors. Immunity (2013) 39:537-47. doi:10 1016/j.immuni.2013.08.026

31. Getts DR, Martin AJ, Mccarthy DP, Terry RL, Hunter ZN, Yap WT, et al. Microparticles bearing encephalitogenic peptides induce T-cell tolerance and ameliorate experimental autoimmune encephalomyelitis. Nat Biotechnol (2012) 30:1217-24. doi:10.1038/nbt.2434

32. De Smet R, Demoor T, Verschuere S, Dullaers M, Ostroff GR, Leclercq G, et al. Beta-Glucan microparticles are good candidates for mucosal antigen delivery in oral vaccination. J Control Release (2013) 172:671-8. doi:10.1016/j.jconrel. 2013.09.007

Received: 17 December 2013; accepted: 27 December 2013; published online: 09 January 2014

Citation: Fric J, Zelante T and Ricciardi-Castagnoli $P$ (2014) Phagocytosis of particulate antigens - all roads lead to calcineurin/NFAT signaling pathway. Front. Immunol. 4:513. doi: 10.3389/fimmu.2013.00513

This article was submitted to Molecular Innate Immunity, a section of the journal Frontiers in Immunology. Copyright (C) 2014 Fric, Zelante and RicciardiCastagnoli. This is an open-access article distributed under the terms of the Creative Commons Attribution License (CC BY). The use, distribution or reproduction in other forums is permitted, provided the original author(s) or licensor are credited and that the original publication in this journal is cited, in accordance with accepted academic practice. No use, distribution or reproduction is permitted which does not comply with these terms. 